\section{International Crisis Group: Patriotic Mobilisation in Russia. Brüssel: ICG, Juli 2018}

Besprochen von Prof. Dr. em. Leo Bamberger, non-resident fellow am Institut für Sicherheitspolitik an der Universität Kiel; E-Mail: bamberger@ispk.uni-kiel.de

https://doi.org/10.1515/sirius-2018-4016

Die hier vorliegende Studie der International Crisis Group befasst sich mit einem Aspekt der Politik der Putin-Administration, der hierzulande wenig Aufmerksamkeit erregt, offenbar, weil er als antiquiert angesehen wird: die Mobilisierung der Öffentlichkeit für patriotische Zwecke. Die Verfasser der Studie stellen fest, dass die entsprechenden Anstrengungen in den vergangenen Jahren signifikant zugenommen haben und dass dabei vor allem die Bedeutung des Militärs für historische und gegenwärtige Leistungen des Landes hervorgehoben werden. Russland sei nicht das einzige Land, welches sich in patriotischer, militärischer Mobilisierung ergehe, aber die zunehmende Militarisierung der Vaterlandsliebe in Russland sei beachtlich und besorgniserregend. Diese Mobilisierung sei das Ergebnis politischer Initiativen und komme zu einer Zeit, wo Russland zunehmend militärische Instrumente zur Förderung seiner Interessen einsetzt.

Die Studie setzt sich mit der Frage auseinander, ob die patriotische Mobilisierung tatsächlich dazu beitrage die innenpolitischen Rahmenbedingungen für eine zunehmend militarisierte und risikobereite russische Außenpolitik zu verbessern. Diese Frage wird im Wesentlichen positiv beantwortet, allerdings machen die Verfasser auch deutlich, dass die Beziehung zwischen außenpolitischem Abenteurertum und patriotischer Mobilisierung komplexer sei als allgemein angenommen. Die Besetzung und Annexion der Krim etwa habe auf einer Welle patriotischer Mobilisierung stattgefunden, der Erfolg habe diese aber denn auch weiterbefördert. Der wachsende Patriotismus, so die Verfasser, sei ein breiter Trend in der russischen Gesellschaft geworden, der die politischen Kosten der Regierung für militärisches Eingreifen im Ausland reduziere.
Die patriotische Mobilisierung der Gesellschaft gehe auf die russische Regierung zurück, werde aber massiv durch Veteranenverbände, patriotische Vereinigungen unterschiedlichen Zuschnitts sowie die russisch-orthodoxe Kirche unterstützt und in immer weitere Kreise der Gesellschaft hineingetragen. Die betreffenden Organisationen werden vom Kreml mit großem finanziellen Aufwand gefördert.

Die Studie reflektiert daraufhin die Bedeutung der patriotischen Mobilisierung in Russland für den Westen. Sie stellt fest, dass in der russischen Propaganda der Westen heute als eine ähnliche Bedrohung dargestellt wird wie das Dritte Reich. Ausgangspunkt ist die Behauptung, dass die Regierung in Kiew faschistisch sei und dass es beim Kampf in der Ostukraine darum gehe eine weitere faschistische Invasion abzuwenden, die implizit noch schlimmer sei als der deutsche Angriff von 1941. Dagegen können westliche Regierung wenig ausrichten. Allerdings sollte versucht werden verschiedene Sektoren der russischen Gesellschaft anzusprechen und andere Narrative einfließen zu lassen. Wie das im Einzelnen geschehen solle, lassen die Verfasser allerdings offen. Sie räumen ein, dass es sehr schwierig sein wird, die legitimen Ziele von ökonomischen Sanktionen des Westens einer russischen Gesellschaft zu erklären, die für patriotische Mobilisierung anfällig ist, weil vor allem die schlechte ökonomische Ausgangslage eine der Ursache für diese Anfälligkeit ist.

Die Studie gelangt zu dem Ergebnis, dass es durchaus sein kann, dass die russische Regierung Gefangener ihrer eigenen Mobilisierungsstrategie wird. Je mehr patriotische Gesinnung und militärischer Aufbruch in einer Gesellschaft dominierend werden, umso größer wird der politische Druck in Krisenzeiten militärische Optionen zu verfolgen. Die Studie empfiehlt der russischen Regierung daher dringend die patriotische Mobilisierung einzustellen. Es ist zu bezweifeln, dass dieser gut gemeinte Rat in Moskau befolgt wird. Vielmehr ist davon auszugehen, dass die patriotische Mobilisierung in Russland weiter um sich greifen und dazu beitragen wird, dass sich das militärische Risikoverhalten der russischen Regierung verschärft.

Patriotische Mobilisierung in Friedenszeiten hat in Russland - darauf weist die Studie leider nicht hin - eine lange Tradition, die bis ins Zarenreich zurückreicht. In der Regel diente sie der Ablenkung von inneren Problemen und hat vor allem zum Ausbruch des Ersten Weltkriegs beigetragen. Anders als 1914 gibt es heute in westlichen Staaten keine vergleichbare, staatlich gelenkte patriotische Mobilisierung. Insofern wird sich der Sommer 1914 nicht wiederholen, aber die Unsicherheit über die Bereitschaft der russischen Regierung mit einseitigen mili- 
tärische Aktionen fait accomplis zu schaffen bleibt ein wesentlicher Anlass zur Sorge.

https://www.crisisgroup.org/europe-central-asia/ caucasus/russianorth-caucasus/251-patriotic-mobilisation-russia 